http://jmscr.igmpublication.org/home/ ISSN (e)-2347-176x ISSN (p) 2455-0450 crossref DOI: https://dx.doi.org/10.18535/jmscr/v10i2.07

\title{
Immediate Effect of Craniosacral Therapy in a Patient with Non-Specific Low Back Pain- A Case Report
}

\author{
Authors \\ Syeda Sameeha Tartil ${ }^{1}$, Sakshi Shah ${ }^{2}$, Dr Shrikant Mhase ${ }^{3}$ \\ ${ }^{1,2}$ Intern, MGM School of Physiotherapy, Aurangabad, Maharashtra, India \\ ${ }^{3}$ Assistant Professor, MGM School of Physiotherapy, Aurangabad, Maharashtra, India
}

\begin{abstract}
Introduction: In the middle-aged population, low back discomfort is a prevalent condition. In her everyday routine, a housewife has a lot of work. The majority of housewives are unaware of ergonomics, which puts them at risk for musculoskeletal illnesses, particularly low back discomfort. Women who have learned to live with distress are riskier. Craniosacral therapy is a manual treatment method that is very light touch and it is used to put patient at ease so help to alleviate various kinds of pain, it is the method which tries to get deep down into the CNS or even beneath it to facilitate movement of cerebrospinal fluid and movement of blood flow and things of the body to improve the body's ability to heal itself.

Case Description: For the past year, a 40-year-old female housewife had been suffering from chronic low back pain and had been treated with physiotherapy and drugs. She went to the Orthopaedic outpatient clinic after experiencing a recent recurrence of discomfort, and she was prescribed physiotherapy. Her normal functions were disturbed.

Procedure: To reduce pain and restore lumbar spine mobility, a procedure called craniosacral treatment was used.

Result: The result was calculated based on the pre-treatment and post-treatment sessions using various outcome measure tools that showed significant changes in the pain intensity and measurement.

Purpose: There is immediate effect of craniosacral therapy for low back pain in a patient with chronic low back pain which aids in pain relief, leading to improved functional activities of daily living and speeding up positive physical therapy outcomes.

Limitations: The results of this case study cannot be extrapolated to a larger sample size because it is a case study. More research is needed to better understand how to choose Craniosacral therapy and how effective it is over time.
\end{abstract}

Keywords: low back pain, craniosacral therapy, case report, housewife.

\section{Introduction}

Low back pain (LBP) is a fairly prevalent complaint accounting for the second most common reason people visit a doctor. LBP can manifest itself in a variety of ways including muscular aches, radiating pain from the back to the legs, and limited range of motion (ROM). ${ }^{1}$
LBP can be caused by a variety of factors, including poor posture, poor biomechanics, falls, and overuse for example, lifting too much weight. Anyone can get low back discomfort, but those who are fat and/or sedentary are at a higher risk. Non-medical and medical therapies are used to treat chronic low back pain. Nonmedical therapies 
include psychological approaches, electrical stimulation, and traction, as well as lumbar stabilisation exercises such as bridging and planking Variation, Nonsteroidalantiinflammatory drugs (NSAIDs) and opioids are used as medical interventions. ${ }^{2}$

This case report describes the Effectiveness of craniosacral therapy in a Housewife with chronic intermittent low back pain, and it will most likely provide evidence for this manual therapy technique. This report will also assist the therapist in choosing a technique that needs active participation from the patient, making him or her a vital part of the therapeutic process. This approach was chosen to provide rapid pain relief and to minimize impairment caused by low back pain.

Craniosacral therapy is a manual treatment method that is very light touch and it is used to put patient at ease so help toalleviate various kinds of pain, it is the method which tries to get deep down into the CNS or even beneath it to facilitate movement ofcerebrospinal fluid and movement of blood flow and things of the body to improve the body's ability to heal itself.

It is different fromtraditional therapy is other techniques require a general amount of force but CST isholistic approach as it involves the body as whole. There is the connection between themind andthe body.

If the patient had stress or chronic pain,anxiety about pain like is it serious how is going to better, when it is going to be better which creates lot of anxiety which trigger the pain spasm feedback loop. Which makes the pain situation worse?

The main factors which help a patient to heal faster is faith on the therapy, andit will only come after the feeling of getting better by the treatment, another factor is taking part in exercise, cardiovascular exerciseregularly which help to improve the fluid flow in cardiovascular system.

\section{Description of Case:}

The subject of this case study was a homemaker who had been suffering from chronic intermittent low back pain for a year. The finger to floor test Measurement, as well as the numerical rating scale for pain, were presented as outcome measures.

\section{History:}

Mrs. Syed, a 40-year-old housewife, has been suffering from low back discomfort for the past year. She went to the Orthopaedic department with a recent recurrence of discomfort and was prescribed NSAIDs as well as physiotherapy treatment.

Examination: Grade 3 local tenderness at L4 and L5 spinous process. She had bilateral Iliopsoas tightness. FABER test was bilaterally positive. The measurement for finger to floor test was $28 \mathrm{~cm}$.

\section{Consent:}

Following the assessment, the therapist explained the findings, the method, and the procedure's requirements. And also requested Her written consent.

Pre therapy scores: Scores of NPRS ${ }^{3}$ for forward Flexion was $8 / 10$ and for floor sitting was $5 / 10$.

Patient position: supine lying position, arm by the side and neck in neutral position.

\section{Procedure:}

the therapist will usually treat you on a coach fully clothed, Patient should be in quiet room, then take the past medical history the treatment session begins with a consultation with your trained therapist to identify a troubled areas, soft music and low lighting are often used to increase your state of relaxation.

The therapist assesses by taking information which is gained in motion testing and by the applications of palpation method that is fluid direction and traction or compression decompression of tissues and tissue structures to assess the existence of possible disruption and or restrictions in your muscles.After proper assessment and getting the knowledge of disturbance in the patient's back the treatment started by asking the patient to close the eyes and take deep breaths and then therapist will apply just 5 grams of pressure on Lumbar vertebrae and then on paraspinal muscles which is the disturbed area of the patient. 
The patient will feelrelaxedand there will be senseof softening in her back. A brief reassessment Was done between each set.

\section{Discussion}

Mrs. Syed had considerable benefits in term of pain relief and spinal mobility. AsinWe have started from L2 vertebra the reason is sympathetic nervous system because it is believed that the system which is responsible for flight, fight, fright of our body which puts the body in stress.

The focus of CST is central placement of hands to assist release of the body is connective tissue orfascia. fascia (band)is covering found throughout the body including organs, glands, nerves, muscles, bloodvessels, brain and spinal cord these covering forms the body wide connective network. CST is based on the theory that controlled movements at the cranial sutures of the skull negatively affect rhythmic impulses transported through the cerebral spinal fluid from the cranium to the sacrum. ${ }^{4}$ and can removes blockages from the normal flow while enhances the body's ability to heal. the hand of therapist gives them a piezoelectric effect to the patient's body.

Craniosacral Therapy diagnoses and treats the craniosacral system by using gentle and precise touches. CST aims to restore sympathetic nerve activity, which is commonly exacerbated in people with chronic pain, by changing craniosacral body rhythms in addition to releasing myofascial structures. ${ }^{5}$ CSTis used to improve the Craniosacral rhythm by biological processes through boosting weren't and self-regulationselfcorrection and self-healing. The Central and Autonomic Nervous Systems are improved through Craniosacral Therapy, which promotes relaxation, well-being, and body homeostasis.

Craniosacral Therapy enhances paratympanic nerve system activity, resulting in a lower heart rate and improved coronary flow, although this alone is not enough to identify an increase or decrease in well-being.
The female benefited psychologically from this touch therapy technique since it provided rapid pain alleviation. She developed a good adherence and compliance to the treatment, as well as a profound relaxation and subsequently recollection of memories and falling asleep throughout the treatment. This therapy, combined with home exercises and ergonomic counselling, could be good for a housewife like her who is busy during the day with all household tasks and cannot make it to the physiotherapy department on a regular basis. Craniosacral therapy is almost risk-free and only takes a few minutes per session. The outcomes of the study imply that, unless a significant illness is present, Craniosacral therapy can be used to treat people with chronic low back pain. Due to the noninvasiveness of the therapy, there are very few adverse side effects, making this treatment in reduction of back pain highly appealing to individuals whoSuffer from chronic pain. With all of the different treatment options of back pain being analyzed in evidence-based Medicine review, patients still seem to experience similar outcomes despite variation in provider, Treatment and cost of treatments. ${ }^{4}$

\section{Conclusion:}

For the immediate relief of persistent intermittent low back pain, craniosacral therapy can be utilised as an adjuvant to traditional physiotherapy. CST is an alternative therapeutic method that aims to relieve constraints surrounding the spinal cord and brain, allowing the body to function normally again.

\section{Limitations and Recommendations:}

The results of this case study cannot be extrapolated to a larger sample size because it is a case study. More research is needed to better understand how to choose Craniosacral therapy and how effective it is over time.

Conflicts of Interests: None Declared. 


\section{References}

1. Maleki, Samir, "Low Back Pain: A Case Study". Physical Therapy Scholarly Projects. (2015); 610. https://commons.und.edu/pt-grad/61

2. Mazreati N, Rahemi Z, Aghajani M, Ajorpaz NM, Mianehsaz E. Effect of craniosacral therapy on the intensity of chronic back pain of nurses: A randomized controlled trial. Nursing Practice Today. 2021 May 24;8(4):313-21.

3. Childs JD, Piva SR, Fritz JM. Responsiveness of the numeric pain rating scale in patients with low back pain. Spine. 2005 Jun 1;30(11):1331-4.

4. Castro-Sanchez AM, Lara-Palomo IC, Mataran-Penarrocha GA, SaavedraHernández M, Pérez-Mármol JM, AguilarFerrándiz ME. Benefits of Craniosacral therapy in patients with chronic low Back pain: a randomized controlled trial. The journal of alternative and complementary medicine. 2016 Aug 1;22(8):650-7.

5. Cutler MJ, Holland BS, Stupski BA, Gamber RG, Smith ML. Cranial manipulation can alter sleep latency and sympathetic nerve activity in humans: a pilot study. Journal of Alternative \& Complementary Medicine. 2005 Feb 1;11(1):103-8.

\section{List of Abbreviations}

CST: Craniosacral therapy

CSF: Cerebrospinal fluid

CNS: Central nervous system

NPRS: Numerical pain rating scale

LBP: Low back pain

NSAIDS: Non-steroidal anti-inflammatory drugs. 